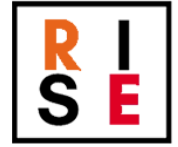

International Journal of Sociology of Education

\section{Hipatia Press}

www.hipatiapress.com

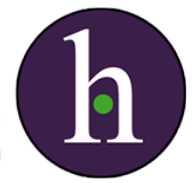

Instructions for authors, subscriptions and further details:

http://rise.hipatiapress.com

\title{
Habilidades Sociales, Autoconcepto y Autoestima en Adolescentes Peruanos de Educación Secundaria
}

Daniel Rubén Tacca Huamán ${ }^{1}$

Renzo Cuarez Cordero ${ }^{2}$

Roberto Quispe Huaycho ${ }^{3}$

1) Universidad Tecnológica del Perú

2) CEPUSM, Perú

3) Colegio Técnico Experimental Jesús Obrero, Perú

Date of publication: October $25^{\text {th }}, 2020$

Edition period: October 2020-February 2021

To cite this article: Tacca Huamán, D.R., Cuarez Cordero, R, \& Quispe Huaycho, R. (2020). Habilidades Sociales, Autoconcepto y Autoestima en Adolescentes Peruanos de Educación Secundaria, International Journal of Sociology of Education, 9(3), 293-324 http://doi.org/10.17583/rise.2020.5186

To link this article: http://dx.doi.org/10.17583/rise.2020.5186

\section{PLEASE SCROLL DOWN FOR ARTICLE}

The terms and conditions of use are related to the Open Journal System and to Creative Commons Attribution License (CC-BY) 


\section{Social Skills, Self-Concept and Self-Esteem in Peruvian High School Students}

Daniel Rubén Tacca Huamán Renzo Cuarez Cordero

Universidad Tecnológica del Perú CEPUSM

Roberto Quispe Huaycho

Colegio Técnico Experimental Jesús Obrero

(Received: 16 February 2020; Accepted: 6 May 2020; Published: 25

October 2020)

\section{Abstract}

The main objective of the research was to know the relationship between social skills, self-concept and self-esteemin Peruvian high school students. The research approach was quantitative with correlational scope and non-experimental- transeccional type. The sample consisted of 324 teenage students from the last two years of high school from different schools in Lima, Peru. The results indicated a positive and medium relationship between social skills and self-concept, the physical, social, personal and sense of control self-concept which have the highest correlation index. A positive and medium relationship between social skills and self-esteem was also found, on the other hand, the relationship between self-concept and self-esteem proved to be large and positive. The evidence showed that men have higher scores in the three variables. Furthermore, adolescents in public schools and those who study with a competency methodology have greater self-concept. No differences were found according to the age of the participants.

Keywords: social skills, selfconcept, self-esteem, teenagers, school 


\section{Habilidades Sociales,}

\section{Autoconcepto y Autoestima en Adolescentes Peruanos de Educación Secundaria}

Daniel Rubén Tacca Huamán

Renzo Cuarez Cordero

Universidad Tecnológica del Perú

CEPUSM

Roberto Quispe Huaycho

Colegio Técnico Experimental Jesús Obrero

(Recibido: 16 Febrero 2019; Aceptado: 6 Mayo 2020; Publicado: 25

Octubre 2020)

\section{Resumen}

El objetivo principal de la investigación fue conocer la relación entre las habilidades sociales, el autoconcepto y la autoestima en estudiantes peruanos de educación secundaria. La investigación fue cuantitativa, correlacional y no experimentaltransversal. La muestra estuvo conformada por 324 estudiantes adolescentes de los dos últimos años de educación secundaria de distintos colegios de Lima, Perú. Los resultados evidencian una relación positiva y mediana entre las habilidades sociales y el autoconcepto, siendo el autoconcepto físico, social, personal y de sensación de control los que presentan mayor índice de correlación. También se encontró una relación positiva y mediana entre las habilidades sociales y la autoestima, por otra parte, la relación entre autoconcepto y autoestima resultó ser grande y positiva. La evidencia muestra que los varones obtienen mayor puntaje en las tres variables. Además, los adolescentes de colegios públicos y que estudian con una metodología con enfoque por competencias presentan mayor autoconcepto. No se encontraron diferencias según la edad de los participantes.

Palabras clave: habilidades sociales, autoconcepto, autoestima, adolescentes, escuela 




1 contexto sociocultural condiciona el desarrollo biopsicosocial del individuo y el establecimiento de ciertas relaciones entre sus miembros; al mismo tiempo, el individuo transforma el entorno social completando un ciclo de interdependencia que se expresa a nivel filogenético, ontogénico y cultural (Patrício et al., 2015). Como explica Tacca (2018), según la teoría sociocultural, el desarrollo cognitivo y de todas las potencialidades del adolescente se debe, especialmente, a las interacciones sociales que se producen en la zona de desarrollo próximo. Como afirma Contini (2015), durante la adolescencia se define la identidad, se busca el contacto con la pareja y con el grupo de pares; de lo anterior, se comprende la importancia de las habilidades sociales en esta etapa del desarrollo humano.

Las habilidades sociales se pueden definir como el conjunto de capacidades especificas e indispensables para interactuar y relacionarse en forma efectiva con los demás (Dávila, 2018); tienen fuertes conexiones con el bienestar personal y la salud integral (Contini, 2015); su desarrollo se contrapone con el carácter individualista y favorecen las competencias emocionales (Gutiérrez \& Expósito, 2015). Estas habilidades incluyen conductas que se aprenden a lo largo de la vida gracias a los modelos de comportamiento propios del contexto donde crece el adolescente. En la actualidad, superan en importancia a las habilidades cognitivas e influyen en la percepción que se tiene de sí mismo (Betancourth et al., 2017). Alvarez (2017) explica que las habilidades se desarrollan e incrementan cuando el sujeto tiene contacto con otros grupos de acción y se establecen en la adolescencia gracias a diversos tipos de aprendizaje (experiencial, por observación, verbal y feedback). La carencia de habilidades sociales facilitaría la aparición de trastornos psicopatológicos, impediría la adecuada consolidación de la identidad y se relacionaría con la deserción escolar (Lacunza \& Contini, 2011).

Betancourth et al. (2017) encontraron que las mujeres adolescentes obtienen puntuaciones significativamente diferentes (más altas) en comparación con sus pares varones respecto a las habilidades sociales. Según Coronel et al. (2011), las adolescentes mujeres de bajo nivel socioeconómico presentaban ansiedad y timidez cuando entablaban relaciones sociales, este comportamiento no se observó en los varones. Muñoz et al. (2008), en un estudio realizado en adolescentes españoles, encontraron que las mujeres tenían más rasgos de sociabilidad que los varones. Malinauskas et al. (2014) 
y García et al. (2014) también encontraron mejores habilidades sociales en las mujeres que en varones. Según Bi et al. (2015), las niñas son más propensas a establecer relaciones positivas con los niños. Por otro lado, Sadovnikova (2016) encontró que las adolescentes rusas se autoperciben tímidas, dependientes y obedientes.

Santana et al. (2018) encontraron que los adolescentes en contextos desfavorables (pobreza, marginación, etc.) presentan escasas estrategias y destrezas socioemocionales, inadaptación social, baja asertividad, conductas agresivas y son propensos al consumo de drogas y alcohol. La familia y el grupo social son espacios de gran influencia en el desarrollo de las habilidades sociales y el autoconcepto (Adarve et al., 2019).

El autoconcepto se refiere al conjunto de rasgos y características que un individuo acepta como parte de sí mismo (Kipp, 2016); es un factor psicosocial importante en la construcción de la personalidad, se ve condicionado por la percepción y las experiencias sociales, físicas $y$ espirituales (Espejo et al., 2018). Las principales funciones que cumple el autoconcepto, según García (2003), son: (1) permite la comprensión personal para un adecuado desempeño social y (2) regula de la conducta. Veas et al. (2019) comentan que existe amplia literatura sobre el estudio del autoconcepto relacionado con la autoestima y el rendimiento académico; también con la inteligencia emocional, la violencia escolar, el consumo de sustancias psicoactivas, satisfacción vital, estilo de vida y sociabilización familiar (Reynoso et al., 2018). Según explica Espejo et al. (2018), el autoconcepto es un constructo complejo que amerita ser estudiado en la adolescencia ya que es una etapa crítica llena de complicaciones por la aparición de nuevas habilidades, interacciones y relaciones psicosociales.

Los estudiantes de educación secundaria que se caracterizan por una actitud positiva frente a los retos, que desarrollan una búsqueda activa de soluciones y que buscan el apoyo de otros para resolver un problema, presentan altos puntajes en la evaluación del autoconcepto académico y habilidades sociales (Morales, 2017). Según Jaureguizar et al. (2018), los sujetos que presentan bajo autoconcepto y carencias en el desarrollo de habilidades sociales presentan menores recursos para afrontar situaciones difíciles. Onetti et al. (2019) no encontraron diferencias en los puntajes del autoconcepto según sexo, a excepción de la dimensión emocional donde las niñas (entre 10 y 14 años) obtienen mayor puntaje; además, se evidenció que 
el autoconcepto obtiene menor puntaje en estudiantes de secundaria en comparación con los de primaria. Guillén y Ramírez (2011) reportaron que los estudiantes con mayor edad y de los últimos años de primaria presentan mayor puntuación en autoconcepto.

Caldera et al. (2018) encontraron una relación significativa entre las habilidades sociales y el autoconcepto; evidenciaron que mientras el autoconcepto se fortalece, se incrementa el desarrollo de las habilidades sociales en los jóvenes. Ballester et al. (2006) resaltan que los adolescentes con mayor autoaceptación presentan mayores estrategias de relaciones interpersonales. Baquerizo y Geraldo (2016) evidenciaron que el autoconcepto académico, social, familiar, físico y emocional se relaciona positivamente con las habilidades sociales; particularmente concluyeron que, a mayor autoconcepto social, mayor desarrollo de las habilidades sociales. En el trabajo realizado por Aqeel (2017), las habilidades sociales de los jóvenes se relacionan positivamente con el conocimiento de sí mismos y el cumplimiento exitoso de actividades laborales y académicas. En el mismo estudio, no se encontraron diferencias en las habilidades sociales entre hombres y mujeres; sin embargo, los hombres mostraron mayores puntajes en el autoconcepto total y en los factores emocional y físico.

Onetti et al. (2019) consideran que el apoyo familiar es importante como agente de socialización; afirman que el autoconcepto familiar favorece la vida social y la independencia del adolescente. Simões y de Castro (2018) encontraron que altos valores de asertividad se relacionan con bajos índices de conductas agresivas, impulsivas o de poca empatía; además, encontraron que los niños con buen índice de sociabilidad presentan alto autoconcepto familiar.

En cuanto a la autoestima, Gámez-Medina et al. (2017) explican que se puede conceptualizar como la autoevaluación que realiza la persona y se traduce en aprobación o rechazo de sí mismo. La autoestima incluye pensamientos y sentimientos sobre la confianza en sí mismo, durante la adolescencia puede cambiar por factores internos o externos (Schoeps et al., 2019). Según Massenzana (2017), la autoestima es una valoración que realiza el sujeto en base a las autopercepciones que tiene sobre si y, estas últimas, se originan gracias a las experiencias vividas (familiares, sociales, físicas, etc.). Para Gatto et al. (2017), la autoestima cumple un rol critico en las relaciones sociales del adolescente. De lo anterior, es posible colegir que la 
autopercepción que construye el individuo está condicionada por el grupo social al cual pertenece; así mismo, el significado emocional y valoración que le atribuye a esas características dependerá del sentido de pertenecía del individuo hacia su grupo social (Tajfel, 1981).

Según Bi et al. (2015), altos niveles de autoestima están asociados con una alta calidad en las relaciones interpersonales. Harris y Orth (2019), luego de un meta-análisis de 48 muestras, concluyeron que existe una relación reciproca entre la autoestima y las relaciones sociales en todas las etapas de la vida, lo que significaría la existencia de un ciclo de retroalimentación entre ambas variables y un respaldo empírico a la teoría del sociómetro, de la valoración reflejada y del apego. La autoestima es un factor mediador entre la relación de pares y el comportamiento social que muestran los adolescentes (Thompson et al., 2016). Bi et al. (2015) encontraron que las relaciones interpersonales del grupo de pares del mismo sexo son más importantes para la autoestima que los otros tipos de relaciones interpersonales. Existe una relación positiva entre la autoestima y las conductas asertivas en los adolescentes (Ayvar, 2017). Según Schoeps et al. (2019), las habilidades emocionales incrementan la autoestima en los adolescentes.

Cheng y Furnham (2017), en un estudio con adolescentes británicos, encontraron que las mujeres presentan menor puntuación en autoestima general en comparación con los varones, sin embargo, las diferencias no fueron significativas. En México, Gámez-Medina et al. (2017) encontraron que los varones presentan mejor autoestima que las mujeres adolescentes. Bleidorn et al. (2016) realizaron un estudio intercultural para examinar las diferencias de la autoestima según el género y la edad, encontrando que los varones presentan mayor nivel de autoestima y que, en ambos sexos, aumenta conforme avanza la edad. Riquelme et al. (2018) reportan que los adolescentes varones presentan mayor vulnerabilidad en la autoestima familiar, las mujeres son más vulnerables en la autoestima emocional; además, varones y mujeres son vulnerables en la autoestima física.

Según Andrade-Salazar et al. (2017) cuando el adolescente no logra resolver los problemas que se presentan puede desmotivarse y frustrarse. Los adolescentes con buena autoestima, pueden afrontar mejor las situaciones estresantes (Thompson et al., 2016). Orgilés et al. (2017), explican que la autoestima se relaciona con el bienestar del adolescente y con la capacidad de usar los recursos cognitivos, emocionales y sociales para adaptase a su 
entorno. Jin-Yeul (2017) encontró una relación positiva y significativa entre las relaciones interpersonales, el apoyo social (familiar, amigos y profesores) y la adaptación a la vida universitaria. Según Cheng y Furnham (2017), los jóvenes que en su infancia presentaron un entorno social-familiar alto, lograron altas puntuaciones en pruebas de capacidad cognitiva, pocos problemas de conducta y autoestima alta. Para Keane y Loades (2016), niveles bajos de autoestima podría traer riesgos a la salud mental de los adolescentes.

Como en la adolescencia se producen cambios a nivel físico, psicológico y social, el estudio de ciertos aspectos de naturaleza cognitiva y socio-afectiva en esta etapa del desarrollo humano resulta importante para conocer, con mayor evidencia empírica, los factores que intervienen en el proceso educativo. Por lo anterior, la presente investigación tiene como objetivo principal conocer la relación entre las habilidades sociales, el autoconcepto y la autoestima en adolescentes peruanos de educación secundaria, así mismo se busca investigar la variación de los resultados según las características sociodemográficas: sexo, edad, tipo de colegio y metodología de enseñanza del centro educativo.

\section{Método}

\section{Diseño de la investigación}

Debido al tratamiento estadístico realizado a los datos, la investigación tiene un enfoque cuantitativo, de alcance correlacional, con un diseño no experimental y transversal (Hernández-Sampieri y Mendoza, 2018).

\section{Participantes}

La muestra estuvo conformada por 324 estudiantes de secundaria de diversos colegios de la ciudad Lima, Perú. Los colegios donde estudian los participantes, pertenecen a un NSE medio. Se tuvo como criterios de inclusión: (1) estudiar en alguno de los dos últimos años de educación secundaria y (2) participar voluntariamente en la investigación. La muestra presentó una edad promedio de 16 años $(D E=0.86)$, con edad mínima y máxima de 15 y 18 años, respectivamente. El $69.1 \%$ de la muestra eran hombres y el $30.9 \%$ mujeres, $72.2 \%$ pertenecían a colegios estatales, mientras 
que el $27.8 \%$ a colegios privados. Además, el $15.4 \%$ pertenecían a colegios con metodología preuniversitaria (solo se desarrollan contenidos conceptuales), mientras que el $84.6 \%$ estudiaban en colegios no preuniversitarios (con metodología por competencias).

\section{Instrumentos.}

Escala de Habilidades Sociales (adaptación peruana realizada por Ruiz, 2006). La escala original fue creada por Elena Gismero Gonzales en Madrid en el año 1997, este instrumento evalúa las habilidades sociales en adolescentes y adultos. El instrumento está compuesto por 33 ítems distribuidos en 6 dimensiones: autoexpresión de situaciones sociales, defensa de los propios derechos como consumidor, expresión de enfado o disconformidad, decir no y cortar interacciones, hacer peticiones e iniciar interacciones positivas con el sexo opuesto (Gismero, 2010). De los 33 Ítems, 28 están redactados en sentido negativo, señalando déficit de habilidades sociales. Además, el instrumento presenta un formato de respuesta tipo Likert de 4 alternativas $(1=$ No me identifico, en la mayoría de veces no me ocurre o no lo haría; $2=$ No tiene que ver conmigo, aunque alguna vez me ocurra; $3=$ Me describe aproximadamente, aunque no siempre actúe así o me sienta así; 4 = Muy de acuerdo, me sentiría así o actuaria así en la mayoría de los casos).

La escala de habilidades sociales fue adaptada al contexto peruano por Ruiz (2006), en la adaptación se comprobó que la escala se organiza en 6 factores al igual que la escala original. Asimismo, tanto en el instrumento original como en la adaptación se obtuvo un coeficiente de consistencia interna alto (.88 y .90 respectivamente). En la presente investigación, se aplicó la prueba de confiabilidad a través del alfa de Cronbach a la escala general y a las seis dimensiones, en la tabla 1 se observa que los valores obtenidos son iguales o superiores a .60, lo que indicaría que el instrumento es confiable. 
Tabla 1

Índices de confiabilidad de la escala de habilidades sociales y sus dimensiones

\begin{tabular}{lc}
\hline & Alfa de Cronbach \\
\hline Escala Total & .79 \\
Autoexpresión de situaciones sociales & .71 \\
Defensa de los propios derechos como consumidor & .64 \\
Expresión de enfado o disconformidad & .61 \\
Decir no y cortar interacciones & .68 \\
Hacer peticiones & .70 \\
Iniciar interacciones positivas con el sexo opuesto & .66 \\
\hline
\end{tabular}

Cuestionario de Autoconcepto de Garley (García, 2001). La escala fue creada por García (2001), con el propósito de evaluar el autoconcepto en niños y adolescentes. Está conformado por 48 ítems distribuidos en 6 dimensiones: física, social, intelectual, familiar, sensación de control, autoevaluación personal. Del total, 11 están redactados de forma inversa. El instrumento presenta un formato de respuesta tipo Likert con 5 opciones $(1=$ nunca, $2=$ pocas veces, $3=$ no sabría decir, $4=$ muchas veces y $5=$ siempre). La escala original obtuvo un coeficiente alfa de Cronbach de .87, lo cual indica una alta confiabilidad del instrumento. Del mismo modo, en la presente investigación se obtuvo un coeficiente de .88 , el cual indica que los puntajes son confiables (ver tabla 2).

Tabla 2

Índices de confiabilidad de la escala de autoconcepto y sus dimensiones

Alfa de Cronbach

\begin{tabular}{ll}
\hline Escala Total & .88 \\
Dimensión física & .75 \\
Dimensión social & .79 \\
Dimensión intelectual & .86 \\
Dimensión familiar & .82 \\
Sensación de Control & .84 \\
Autoevaluación personal & .74 \\
\hline
\end{tabular}




\section{Tacca, Cuarez \& Quispe - Habilidades Sociales}

Cuestionario de Autoestima (adaptación argentina realizada por Góngora y Casullo, 2009). La escala original fue creada por Rosenberg en 1965, la cual evalúa los sentimientos de respeto y aceptación de sí mismo como medio para denotar valía y satisfacción. Está compuesta de 10 ítems, de los cuales 5 de ellos están redactados en forma negativa (ítems: 2, 5, 8, 9 y 10). Los ítems presentan un formato Likert de 4 puntos $(1=$ muy en desacuerdo y $4=$ muy de acuerdo).

La adaptación al español fue realizada por Atienza et al. (2000) en una muestra de adolescentes españoles. La prueba de validez mostró una estructura unidimensional para el instrumento, además los autores evaluaron la confiabilidad a través del método test-retest. Góngora y Casullo (2009) realizaron la adaptación argentina del instrumento en población general $(n=313)$ y clínica $(n=60)$, se encontró que el instrumento se ajustaba a un modelo de una sola dimensión en ambos grupos y se obtuvo índices de confiabilidad adecuados (alfa de Cronbach de .70 y .78 respectivamente). En la presente investigación se obtuvo alfa de Cronbach de .75, lo cual muestra la confiabilidad del instrumento.

\section{Procedimiento}

La muestra fue no probabilística: muestreo por conveniencia; este tipo de muestreo permite al investigador tomar en cuenta los casos disponibles a los que tiene acceso (Hernández-Sampieri y Mendoza, 2018). Primero se envió el consentimiento informado a los padres de 348 adolescentes, en este documento se indicó los objetivos de la investigación, la duración de las pruebas, el derecho del adolescente de retirarse en el momento que desee; también se informó sobre la participación anónima y el uso de los datos únicamente para los objetivos del estudio. 338 padres autorizaron que sus hijos participen en el estudio. Tras la aplicación, 14 casos no fueron tomados en cuenta debido a que no completaron los cuestionarios, por lo cual se quedó con una muestra de 324 estudiantes. Además de los cuestionarios, los participantes completaron una ficha sociodemográfica, la cual permitió obtener información tal como: sexo, edad, tipo de colegio (privado y público) y metodología de enseñanza (preuniversitario y no preuniversitario). El llenado de los documentos duró 36 minutos aproximadamente por persona y 
se realizó en las aulas de los colegios. La recolección de datos se llevó a cabo en el último trimestre del año escolar.

\section{Análisis de Datos}

Para analizar los datos se utilizó el paquete estadístico SPSS Statistics versión 22.0. Primero se realizó un análisis de identificación de casos atípicos, se encontraron tres casos con puntuaciones que se alejaban extremadamente de la media. A pesar de lo hallado, se decidió incluirlos en los análisis debido a dos razones: (1) los casos formaban parte de la diversidad de respuesta de la muestra y (2) que no generaran variación significativa en los resultados. Se realizó la prueba Shapiro-Wilk para evaluar la normalidad de las puntuaciones obtenidas en cada prueba (ver tabla 3 ). Se encontró falta de normalidad en todas las puntuaciones, excepto en la dimensión física del autoconcepto.

Tabla 3

Resultados de prueba de normalidad: Shapiro-Wilk

\begin{tabular}{clcccc}
\hline & & $M$ & $D E$ & W & Sig. \\
\hline & $\begin{array}{l}\text { Autoexpresión de situaciones } \\
\text { sociales }\end{array}$ & 23.07 & 4.31 & .99 & .003 \\
& $\begin{array}{l}\text { Defensa de los propios } \\
\text { derechos como consumidor }\end{array}$ & 13.71 & 2.73 & .99 & .002 \\
$\begin{array}{c}\text { Escala de } \\
\text { habilidades } \\
\text { sociales }\end{array}$ & $\begin{array}{l}\text { Expresión de enfado o } \\
\text { disconformidad }\end{array}$ & 10.63 & 2.53 & .98 & .001 \\
& Decir no y cortar interacciones & 16.65 & 3.53 & .99 & .002 \\
& Hacer peticiones & 14.33 & 2.69 & .98 & .000 \\
& Iniciar interacciones positivas & 13.88 & 2.82 & .98 & .001 \\
\hline \multirow{5}{*}{ con el sexo opuesto } & Dimensión física & 27.00 & 5.63 & .99 & .081 \\
& Dimensión social & 26.05 & 5.08 & .99 & .015 \\
& Dimensión familiar & 28.12 & 5.02 & .98 & .004 \\
& Dimensión intelectual & 26.35 & 4.77 & .99 & .014 \\
& Autoevaluación personal & 28.89 & 5.29 & .98 & .002 \\
& Sensación de control & 26.58 & 5.65 & .91 & .000 \\
\hline Autoestima & & 32.28 & 4.00 & .97 & .008 \\
\hline
\end{tabular}




\section{Tacca, Cuarez \& Quispe - Habilidades Sociales}

En el análisis de los coeficientes de asimetría $(<|3|)$ y curtosis $(<|8|)$, se concluyó que no existía falta de normalidad severa (Kline, 2005), con estos resultados se emplearon los análisis paramétricos correspondientes. Se realizó el análisis correlacional con el coeficiente $r$ de Pearson entre las variables de estudio. Por último, se realizaron contrastes de medias utilizando la prueba paramétrica $t$ de Student según las características sociodemográficas.

\section{Resultados}

A nivel descriptivo, se obtuvo como puntaje promedio 92.28 en la escala total de habilidades sociales (ver Tabla 4).

Tabla 4

Resultados descriptivos de la escala habilidades sociales

\begin{tabular}{lcccc}
\hline & $M$ & $D E$ & Mínimo & Máximo \\
\hline Total habilidades sociales & 92.28 & 12.37 & 61 & 126 \\
$\begin{array}{l}\text { Autoexpresión de situaciones sociales } \\
\text { Defensa de los propios derechos como }\end{array}$ & 23.07 & 4.31 & 10 & 32 \\
$\begin{array}{l}\text { consumidor } \\
\text { Expresión de enfado o disconformidad }\end{array}$ & 13.71 & 2.73 & 6 & 20 \\
$\begin{array}{l}\text { Decir no y cortar interacciones } \\
\text { Hacer peticiones }\end{array}$ & 10.63 & 2.53 & 4 & 18 \\
$\begin{array}{l}\text { Iniciar interacciones positivas con el sexo } \\
\text { opuesto }\end{array}$ & 14.33 & 3.53 & 6 & 24 \\
\hline
\end{tabular}

Con relación a la variable autoconcepto, se obtuvo como media un puntaje de 162.73 (ver Tabla 5). 
RISE - International Journal of Sociology of Education, 9(3) 305

Tabla 5

Resultados descriptivos de la escala autoconcepto

\begin{tabular}{lcccc}
\hline & $M$ & $D E$ & Mínimo & Máximo \\
\hline Total Autoconcepto & 162.73 & 23.96 & 100 & 242 \\
Dimensión física & 27.00 & 5.63 & 8 & 40 \\
Dimensión social & 26.05 & 5.08 & 11 & 39 \\
Dimensión familiar & 28.12 & 5.02 & 16 & 40 \\
Dimensión intelectual & 26.35 & 4.77 & 13 & 39 \\
Dimensión personal & 28.89 & 5.29 & 12 & 40 \\
Sensación de control & 26.58 & 5.65 & 10 & 62 \\
\hline
\end{tabular}

La variable autoestima obtuvo 32.28 como promedio global (ver tabla 6).

Tabla 6

Resultados descriptivos de la escala autoestima

\begin{tabular}{cccc}
\hline$M$ & $D E$ & Mínimo & Máximo \\
\hline 32.28 & 4.00 & 21 & 39 \\
\hline
\end{tabular}

Para responder el objetivo principal, se correlacionó las variables habilidades sociales, autoconcepto y autoestima, encontrándose relaciones positivas. Siendo las correlaciones entre habilidades sociales-autoconcepto y habilidades sociales-autoestima de tamaño mediano, mientras que la relación entre autoconcepto-autoestima fue un tamaño grande (ver tabla 7).

Tabla 7

Correlaciones entre las variables de estudio

\begin{tabular}{lccc}
\hline & 1 & 2 & 3 \\
\hline 1. Habilidades sociales & - & & \\
2. Autoconcepto & $.34^{*}$ & - & \\
3. Autoestima & $.41^{*}$ & $.51^{*}$ & - \\
\hline${ }^{*}<.001$ & & &
\end{tabular}


En la tabla 8 se aprecia las correlaciones entre las dimensiones del autoconcepto con las habilidades sociales y la autoestima. Se observa que las relaciones entre las habilidades sociales y las dimensiones del autoconcepto son de tamaño pequeño a mediano; por otro lado, todas las correlaciones entre autoestima y las dimensiones del autoconcepto, son de tamaño mediano.

Tabla 8

Correlaciones entre habilidades sociales, autoestima y dimensiones del autoconcepto

\begin{tabular}{lcc}
\hline & Habilidades sociales & Autoestima \\
\hline Dimensión física & $.32^{*}$ & $.43^{*}$ \\
Dimensión social & $.30^{*}$ & $.47^{*}$ \\
Dimensión familiar & $.29^{*}$ & $.37^{*}$ \\
Dimensión intelectual & $.24^{*}$ & $.37^{*}$ \\
Dimensión personal & $.31^{*}$ & $.38^{*}$ \\
Sensación de control & $.30^{*}$ & $.33^{*}$ \\
\hline${ }^{*} p<.001$ & &
\end{tabular}

Se encontró correlaciones significativas y positivas entre el autoconcepto y las dimensiones de habilidad sociales: autoexpresión de situaciones sociales, decir no y cortar interacciones, hacer peticiones e iniciar interacciones positivas con el sexo opuesto; siendo estas de tamaño pequeño a mediano. En relación a la autoestima, esta variable presenta relaciones significativas y de tamaño pequeño a mediano con las seis dimensiones de las habilidades sociales (tabla 9). 
Tabla 9

Correlaciones entre autoconcepto, autoestima y las dimensiones de las habilidades sociales

\begin{tabular}{lcc}
\hline & Autoconcepto & Autoestima \\
\hline Autoexpresión de situaciones sociales & $.37^{* *}$ & $.38^{* *}$ \\
Defensa de los propios derechos como consumidor & .13 & $.15^{*}$ \\
Expresión de enfado o disconformidad & .09 & $.21^{* *}$ \\
Decir no y cortar interacciones & $.16^{*}$ & $.22^{* *}$ \\
Hacer peticiones & $.21^{* *}$ & $.31^{* *}$ \\
Iniciar interacciones positivas con el sexo opuesto & $.31^{* *}$ & $.29^{* *}$ \\
\hline${ }^{*}<.01, * * \mathrm{p}<.001$ & &
\end{tabular}

También se realizaron comparaciones de medias de acuerdo a las características sociodemográficas de los participantes (sexo, edad, tipo de colegio y metodología de enseñanza). En relación a la variable habilidades sociales, solo se encontraron diferencias significativas entre la característica sociodemográfica sexo, donde se aprecia que los hombres presentan mayores puntajes a comparación de las mujeres (ver tabla 10).

Tabla 10

Prueba t de la variable habilidades sociales según la característica sexo

\begin{tabular}{|c|c|c|c|c|c|c|}
\hline & \multicolumn{2}{|c|}{$\begin{array}{l}\text { Hombres } \\
(n=224)\end{array}$} & \multicolumn{2}{|c|}{$\begin{array}{l}\text { Mujeres } \\
(n=100)\end{array}$} & \multirow[t]{2}{*}{$t$} & \multirow[t]{2}{*}{$d$} \\
\hline & $M$ & $D E$ & $M$ & $D E$ & & \\
\hline Total habilidades sociales & 93.49 & 12.16 & 89.57 & 12.485 & $2.66 * *$ & 0.32 \\
\hline $\begin{array}{l}\text { Autoexpresión de situaciones } \\
\text { sociales }\end{array}$ & 23.59 & 4.09 & 21.89 & 4.581 & $3.34 * *$ & 0.39 \\
\hline $\begin{array}{l}\text { Defensa de los propios } \\
\text { derechos como consumidor }\end{array}$ & 13.68 & 2.66 & 13.79 & 2.900 & -0.34 & 0.04 \\
\hline $\begin{array}{l}\text { Expresión de enfado o } \\
\text { disconformidad }\end{array}$ & 10.63 & 2.51 & 10.65 & 2.607 & -0.08 & 0.01 \\
\hline $\begin{array}{l}\text { Decir no y cortar } \\
\text { interacciones }\end{array}$ & 16.79 & 3.49 & 16.36 & 3.628 & 1.00 & 0.04 \\
\hline Hacer peticiones & 14.70 & 2.61 & 13.51 & 2.706 & $3.76^{* * *}$ & 0.45 \\
\hline $\begin{array}{l}\text { Iniciar interacciones positivas } \\
\text { con el sexo opuesto }\end{array}$ & 14.11 & 2.93 & 13.37 & 2.485 & $2.33^{*}$ & 0.27 \\
\hline
\end{tabular}


En la variable autoconcepto, se encontraron diferencias entre las características sociodemográficas: sexo, tipo de colegio y metodología de enseñanza. Se halló que los participantes hombres presentan un mayor puntaje a comparación de las mujeres (tabla 11). Además, los alumnos de colegio estatal presentan mayores puntajes que los alumnos de colegios privados (tabla 12). Del mismo modo, se encontró que aquellos que estudian bajo la metodología de enseñanza no preuniversitaria muestran puntajes más altos que aquellos bajo la modalidad preuniversitaria (tabla 13).

Tabla 11

Prueba t de la variable autoconcepto según la característica sexo

\begin{tabular}{|c|c|c|c|c|c|c|}
\hline & \multicolumn{2}{|c|}{$\begin{array}{l}\text { Hombres } \\
(n=224)\end{array}$} & \multicolumn{2}{|c|}{$\begin{array}{l}\text { Mujeres } \\
(n=100)\end{array}$} & \multirow[t]{2}{*}{$t$} & \multirow[t]{2}{*}{$d$} \\
\hline & $M$ & $D E$ & $M$ & $D E$ & & \\
\hline $\begin{array}{l}\text { Total } \\
\text { autoconcepto }\end{array}$ & 166.60 & 23.17 & 154.06 & 23.543 & $4.48 * * *$ & 0.54 \\
\hline $\begin{array}{l}\text { Dimensión } \\
\text { física }\end{array}$ & 27.65 & 5.33 & 25.56 & 6.032 & $3.13 * *$ & 0.37 \\
\hline $\begin{array}{l}\text { Dimensión } \\
\text { social }\end{array}$ & 26.51 & 4.98 & 25.00 & 5.156 & $2.50 * *$ & 0.3 \\
\hline $\begin{array}{l}\text { Dimensión } \\
\text { familiar }\end{array}$ & 28.83 & 4.88 & 26.51 & 4.982 & $3.93 * * *$ & 0.47 \\
\hline $\begin{array}{l}\text { Dimensión } \\
\text { Intelectual }\end{array}$ & 26.79 & 4.77 & 25.38 & 4.649 & $2.47 *$ & 0.3 \\
\hline $\begin{array}{l}\text { Dimensión } \\
\text { personal }\end{array}$ & 29.75 & 5.00 & 26.96 & 5.446 & $4.51 * * *$ & 0.53 \\
\hline $\begin{array}{l}\text { Sensación de } \\
\text { control }\end{array}$ & 27.07 & 5.37 & 25.49 & 6.127 & $2.34^{*}$ & 0.27 \\
\hline
\end{tabular}


RISE - International Journal of Sociology of Education, 9(3) 309

Tabla 12

Prueba t de la variable autoconcepto según la característica tipo de colegio

\begin{tabular}{|c|c|c|c|c|c|c|}
\hline & \multicolumn{2}{|c|}{$\begin{array}{c}\text { Estatal } \\
(n=234)\end{array}$} & \multicolumn{2}{|c|}{$\begin{array}{l}\text { Privado } \\
(n=90)\end{array}$} & \multirow[t]{2}{*}{$t$} & \multirow[t]{2}{*}{$d$} \\
\hline & $M$ & $D E$ & $M$ & $D E$ & & \\
\hline Total autoconcepto & 163.45 & 23.88 & 153.75 & 23.639 & 1.96 & 0.41 \\
\hline Dimensión física & 27.11 & 5.58 & 25.67 & 6.162 & 1.21 & 0.25 \\
\hline Dimensión social & 26.15 & 5.06 & 24.75 & 5.169 & 1.30 & 0.27 \\
\hline Dimensión familiar & 28.39 & 4.99 & 24.67 & 4.188 & $3.56 * * *$ & 0.81 \\
\hline $\begin{array}{l}\text { Dimensión } \\
\text { Intelectual }\end{array}$ & 26.36 & 4.75 & 26.21 & 5.124 & 0.15 & 0.03 \\
\hline Dimensión personal & 29.11 & 5.28 & 26.13 & 4.749 & $2.68 * *$ & 0.59 \\
\hline Sensación de control & 26.60 & 5.69 & 26.33 & 5.223 & 0.22 & 0.05 \\
\hline
\end{tabular}

$*<.05 ; * *<.01 ; * * *<.001$

Tabla 13

Prueba t de la variable autoconcepto según la característica metodología de enseñanza

\begin{tabular}{lcccccc}
\hline & \multicolumn{7}{c}{$\begin{array}{c}\text { Pre-universitario } \\
(n=50)\end{array}$} & \multicolumn{2}{c}{$\begin{array}{c}\text { No pre- } \\
\text { universitario } \\
(n=274)\end{array}$} & $t$ & $d$ \\
& $M$ & $D E$ & $M$ & $D E$ & & \\
\hline Total autoconcepto & 154.88 & 21.30 & 164.16 & 24.174 & $-2.54^{* *}$ & 0.41 \\
Dimensión física & 26.10 & 5.53 & 27.17 & 5.642 & -1.24 & 0.19 \\
Dimensión social & 24.92 & 4.96 & 26.25 & 5.079 & -1.71 & 0.26 \\
Dimensión familiar & 26.04 & 4.54 & 28.50 & 5.021 & $-3.46^{* *}$ & 0.51 \\
Dimensión & 25.60 & 5.10 & 26.49 & 4.702 & -1.21 & 0.18 \\
Intelectual & & & & & & \\
Dimensión personal & 27.12 & 5.02 & 29.21 & 5.283 & $-2.59^{* *}$ & 0.41 \\
Sensación de control & 26.78 & 4.61 & 26.55 & 5.827 & -0.27 & 0.04 \\
\hline$*<.05 ; * *<.01 ; * * * .001$ & & & & &
\end{tabular}

En la variable autoestima, solo se encontró diferencias significativas en la característica sociodemográfica sexo, en donde los hombres presentan mayores puntajes que las mujeres (ver tabla 14). 


\section{Tacca, Cuarez \& Quispe - Habilidades Sociales}

Tabla 14

Prueba t de la variable autoestima según la característica metodología de enseñanza

\begin{tabular}{lccccccc}
\hline & \multicolumn{2}{c}{ Hombres } & \multicolumn{2}{c}{ Mujeres } \\
& \multicolumn{2}{c}{$(n=224)$} & \multicolumn{2}{c}{$(n=100)$} & $t$ & $d$ \\
$M$ & $D E$ & $M$ & $D E$ & & \\
\hline $\begin{array}{l}\text { Total } \\
\text { autoconcepto }\end{array}$ & 32.92 & 3.79 & 30.83 & 4.090 & $4.48^{* *}$ & 0.41 \\
\hline$*<.05 ; * * .01 ; * * *<.001$ & & & & &
\end{tabular}

\section{Discusión y Conclusiones}

Según los resultados obtenidos en la muestra de estudio, se ha observado que las habilidades sociales se encuentran en un nivel alto de desarrollo. Lo anterior coincide con los hallazgos de Betancourth et al. (2017) en adolescente colombianos, con el estudio en adolescentes chilenos de Oyarzún et al. (2012) y parcialmente con los resultados de Caballero et al. (2018) sobre el déficit social (retraimiento y ansiedad social) en adolescentes argentinos de bajo nivel socioeconómico. Por otra parte, los resultados parece que no coinciden del todo con los hallazgos de Jaimes et al. (2019) en estudiantes adolescentes de un centro preuniversitario de Perú. En este último trabajo, se registró un puntaje medio de desarrollo en todas las habilidades sociales, lo que indicaría que la muestra estudiada podría presentar ciertos inconvenientes en la integración a la vida académica, comprometiendo su aprendizaje. Es posible, como se analizará más adelante, que las diferencias en el enfoque didáctico de la institución puede afectar el desarrollo de ciertas habilidades en los estudiantes.

En general, los resultados obtenidos en las habilidades sociales distan de los hallazgos de Santana et al. (2018) en adolescentes que viven en contextos desfavorables; es posible que estas diferencias se deban a que las muestras pertenecen a niveles socioeconómicos diferentes. Como la dinámica familiar y el grupo social son espacios que influyen en el desarrollo de estas habilidades (Adarve et al., 2019), es probable que en un contexto desfavorable los adolescentes presenten diversos problemas de índole psicológico, conductual y académico (Lacunza \& Contini, 2011). 
En la presente investigación, las habilidades de autoexpresión, decir no y hacer peticiones obtuvieron el mayor puntaje; estos valores coinciden parcialmente con los hallados por Núñez et al. (2018) ya que, según el reporte estadístico, las habilidades sociales que obtienen mayor puntaje en adolescentes son autoexpresión, defensa de los derechos y hacer peticiones. Los valores reportados en autoexpresión indicarían que los adolescentes de secundaria, en concordancia con lo propuesto por Dávila (2018), estarían en la capacidad de expresarse en forma autónoma y espontánea en diversos contextos. Los resultados en la habilidad de decir no indicarían que están en la capacidad de cortar cualquier comunicación o vínculo que no contribuye a su bienestar o integridad, lo anterior se relacionaría con los planteamientos de Contini (2015). El puntaje en la dimensión hacer peticiones reflejaría la facilidad con la que los jóvenes solicitan ayuda a los demás; lo anterior, según Santana et al. (2018), se debería a que en la adolescencia se amplía el grupo de pares y se aceptan, interesada o desinteresadamente, nuevas amistades de diferente sexo; esto también coincidiría con lo propuesto por Alvarez (2017).

Por otro lado, las habilidades de defensa y expresión del enfado, son las que menor puntaje presentan; esto indicaría que los participantes necesitan mejorar en su comportamiento asertivo frente a desconocidos y al momento de reclamar sus derechos en un contexto de compra o consumo de productos; además, no expresarían adecuadamente su enfado o desacuerdo. Es posible que estos resultados sean reflejo del proceso de maduración neuronal y cognitivo del adolescente. Como explica Tacca (2016), durante la adolescencia, la corteza prefrontal es una de las estructuras que más se demora en madurar, por lo que la inhibición y regulación de ciertas conductas y expresiones (miedo, ira, enfado, etc.) estaría en vías de desarrollo. El proceso heterocrónico de maduración cerebral no solo explicaría los comportamientos e interacciones sociales de los adolescentes, también influiría en el procesamiento de la información y el pensamiento (Moreira \& Bolívar, 2019). El desarrollo cerebral también tendría relación con el desarrollo de las funciones cognitivas (Viejo \& Ortega-Ruiz, 2015) y como las habilidades sociales influyen en los resultados académicos (Coy \& Martin, 2017), es recomendable que ambos aspectos sean considerados por los docentes en la planificación, desarrollo, interacción y evaluación de los aprendizajes.

En cuanto al autoconcepto, se evidencia que las dimensiones con mayor puntaje son el autoconcepto personal y familiar, coincidiendo con la propuesta 


\section{Tacca, Cuarez \& Quispe - Habilidades Sociales}

de García (2003). Esto indicaría que los adolescentes poseen una adecuada valoración general de su persona, presentan predisposición a sentirse satisfechos con la situación actual de su familia, poseen buena comunicación abierta familiar (León-Moreno \& Musitu-Ferrer, 2019) y se sienten libres de compartir experiencias con los miembros de su familia (Malca-Romero \& Rivera-Jiménez, 2019). Por otra parte, es importante mencionar que la sensación de control y el autoconcepto social son las dimensiones con menor puntaje. Lo anterior se traduciría en una sensación de poco control de la situación que viven y presentarían algunas dificultades para sentirse parte del grupo de amigos. Es necesario recordar que la adolescencia es una etapa crítica (Espejo et al., 2018) y que los jóvenes pasan por un proceso heterogéneo de madurez vocacional (García et al., 2017); por ello, es posible que los puntajes obtenidos en estas dos últimas dimensiones reflejen la incertidumbre y una latente preocupación por la elección de la profesión u oficio, la pareja o la aceptación social.

Los resultados descriptivos de la variable autoestima muestran que los adolescentes presentan alto nivel de autoestima, coincidiendo con los hallazgos de Estrada et al. (2019) en estudiantes peruanos, pero no con la autoestima normal reportada por Marchant, et al. (2017) en escolares chilenos. Las puntuaciones obtenidas permiten afirmar que, en general, la muestra de estudio se evalúa positivamente en diversos aspectos como el respeto a sí mismo, la aceptación de "virtudes" y "debilidades", mostrarían cierta satisfacción con las cualidades que poseen y con lo que pueden aportar al grupo social. En el ámbito educativo, estos resultados se podrían traducir en motivación permanente para aprender, asumir responsabilidad sobre sus actos, mostrar conductas sociales y empáticas con los demás, capacidad para resolver controversias en el grupo, ser autocritico con el trabajo académico, etc.

En cuanto al objetivo principal de la investigación, los resultados estadísticos correlacionales muestran una relación positiva y mediana entre las habilidades sociales y el autoconcepto en general. Esto parece coincidir con lo reportado por Onetti et al. (2019); Simões y de Castro (2018); Caldera et al. (2018); Betancourth et al. (2017); Aqeel (2017); Morales (2017); Baquerizo y Geraldo (2016) y Ballester et al. (2006). En este sentido, la evidencia parece indicar que las habilidades sociales son un factor importante, en la construcción del autoconcepto de los adolescentes y viceversa; ambas 
variables contribuyen al desarrollo o no de la otra durante esta etapa. En base a lo anterior, con la promoción de las habilidades sociales a través de actividades didácticas como el juego de roles, el modelado, la coevaluación o la retroalimentación grupal, entre otras; se podría promover un buen ajuste psicosocial y desarrollar un autoconcepto positivo. Por otra parte, no se debe olvidar el papel que juega la familia y las amistades en la interacción de estos dos constructos (Adarve et al., 2019).

El autoconcepto en general presenta una correlación positiva y mediana con las habilidades de autoexpresión e iniciar interacciones con los demás. Lo anterior parece coincidir con los aportes de Contini (2015) y el bienestar personal; Gutiérrez y Expósito (2015) y el rol de las habilidades socioemocionales; Betancourth et al. (2017) y el papel de las habilidades sociales en la percepción que se tiene de uno mismo; y Jaureguizar et al. (2018) y la importancia de las interacciones sociales para afrontar dificultades. En la misma línea, se ha evidenciado que el autoconcepto físico, social, personal y de sensación de control presentan correlaciones medianas con las habilidades sociales. El autoconcepto no es algo que aparece de un momento a otro, es una percepción que se construye a lo largo del desarrollo humano y, particularmente, en la adolescencia se va consolidando como pilar de la personalidad (Espejo et al., 2018). La construcción de una imagen positiva, contribuiría a que los adolescentes entablen comunicación interpersonal con sus pares, posean mayor posibilidad de desarrollar habilidades blandas y aumentaría sus posibilidades de éxito en la vida.

Por otra parte, el autoconcepto familiar e intelectual presentan correlaciones pequeñas con las habilidades sociales, esto no coincidiría con la propuesta de Onetti et al. (2019) y Simões y de Castro (2018). A pesar de reportarse relaciones pequeñas, en general, los adolescentes no mostraron problemas de sociabilidad con los pares y el autoconcepto familiar mostró uno de los índices más altos. Cuando el adolescente crece y va terminando el periodo escolar, es común que se aleje del entorno familiar (sin llegar a rechazarlo) y los lazos con sus pares cobran relevancia en su crecimiento socioemocional. Las relaciones interpersonales con los pares contribuyen a la comprensión de las convenciones sociales y al crecimiento personal. En cuanto al autoconcepto intelectual, es posible que esta dimensión no se vincule estrechamente con las habilidades sociales ya que está más orientado al plano cognitivo e intelectual. 


\section{Tacca, Cuarez \& Quispe - Habilidades Sociales}

Se ha encontrado que la relación entre habilidades sociales y autoestima es positiva y mediana, esto coincidiría con Harris y Orth (2019), Ayvar (2017), Gatto et al. (2017), Orgilés et al. (2017), Thompson et al. (2016), Bi et al. (2015) y Tajfel (1981). Además, los resultados reflejan que el autoconcepto y la autoestima se relacionan también en forma positiva pero grande, concordando con las propuestas de Massenzana (2017) y Tajfel (1981). Los resultados permiten observar cómo estos tres constructos se ligan y el estudio de su interacción puede enriquecer el entendimiento del desarrollo cognitivo, afectivo y social del estudiante adolescente y sus posibles repercusiones en el rendimiento académico. Si la oferta formativa pública y privada promueve el desarrollo de habilidades socio-afectivas en los estudiantes adolescentes, estaría contribuyendo a la formación de un autoconcepto positivo y también ayudarían a mejorar la autoestima de los estudiantes. Lo anterior se traduciría en la formación de personas con capacidad de adaptación (Jin-Yeul, 2017), de resolución de problemas, manejo del estrés y de la frustración (AndradeSalazar et al., 2017; Thompson et al., 2016); además, se reduciría el índice de deserción escolar (Lacunza \& Contini, 2011) y el riesgo de padecer alguna enfermedad mental (Keane \& Loades, 2016; Lacunza \& Contini, 2011).

Los resultados indican que existe diferencia significativa en las habilidades sociales entre varones y mujeres, siendo los primeros quienes obtienen mayor puntaje en la escala total y en las habilidades de autoexpresión, hacer peticiones e iniciar interacciones con el sexo opuesto. Parece que esto coincide con Coronel et al. (2011) y no con Betancourth et al. (2017), Aqeel (2017), Malinauskas et al. (2014), García et al. (2014), Bi et al. (2015) y Muñoz et al. (2008). En cuanto al autoconcepto y la autoestima, en ambos análisis se observó diferencia significativa según el sexo, siendo nuevamente los varones quienes obtienen una ventaja numérica sobre las mujeres. Estos resultados no coincidirían con Cheng y Furnham (2017), pero si con Aqeel (2017), GámezMedina et al. (2017) y Bleidorn et al. (2016). Estos hallazgos se deberían a la interdependencia entre el adolescente y su entorno social (Patrício et al., 2015; Tacca, 2018), es decir, los diversos resultados obtenidos por distintos investigadores se deberían al contexto estudiado, donde influyen directamente las ventajas y desventajas culturales y sociales propias de cada ciudad, de cada país.

Si bien la sociedad peruana, con una idiosincrasia machista por tradición, intenta cambiar las costumbres que otorgan ventajas a los varones sobre las 
mujeres, aún no termina de superar los roles tradicionales de género. Las mujeres adolescentes, como lo explican Caldera et al. (2018), siguen sufriendo los estragos de la frustración y presión social producidos por los estereotipos de belleza, los que afectan su desarrollo social y la construcción de su autoconcepto. En el mismo sentido, Videra-García y Reigal-Garrido (2013) explican que las adolescentes presentan mayor correlación entre el autoconcepto físico y la satisfacción vital, esto significaría que aún en estos tiempos las percepciones físicas juegan un rol importante en la construcción del autoconcepto y autoestima en las adolescentes mujeres. Es posible que esta brecha se mantenga si no se adoptan políticas de estado claras para promover una educación de calidad y con igualdad de derechos $\mathrm{y}$ oportunidades para todos.

En cuanto al tipo de colegio y la metodología de enseñanza, los resultados señalan que los adolescentes de colegios estatales y con metodología no preuniversitaria (enfocada en el logro de competencias) presentan mayor puntaje en el autoconcepto general. En el Perú, el sistema educativo público es muy criticado por las carencias que presenta en cuanto a infraestructura y formación continua de los profesores; sin embargo, la evidencia indicaría que no todo está perdido. Los centros de educación pública se caracterizan por un sistema de aprendizaje basado en el logro de competencias y en los últimos años han implementado una propuesta educativa que integra el aspecto cognitivo, social y afectivo.

Por el contrario, las instituciones privadas con metodología preuniversitaria, en su mayoría, se centran en el desarrollo secuencial de contenidos enciclopédicos con el único objetivo de superar el examen de admisión de una universidad; como afirma Jaimes et al. (2019), es común que los centros preuniversitarios presenten limitaciones en su oferta formativa. Esta diferencia en el enfoque educativo podría ser una explicación del porqué los estudiantes de estos colegios presentan mejores puntajes a pesar de los problemas en infraestructura y de las condiciones socioeconómicas. Como explica Mori (2018), el colegio, por las horas de permanencia de los estudiantes, se debería convertir en un escenario que estimule el desarrollo humano. Una educación holística, que forma personas y ciudadanos, puede contribuir a la construcción de un autoconcepto positivo.

Como el alcance de la presente investigación fue correlacional, la asociación estadística de las variables solo explica las relaciones descritas en 


\section{Tacca, Cuarez \& Quispe - Habilidades Sociales}

el presente informe. Debido a esto, la evidencia no es concluyente, es decir, no se puede hablar de causalidad entre las habilidades sociales, el autoconcepto y la autoestima. Otra limitación del estudio fue su naturaleza transversal: solo se ha evaluado las variables en un momento determinado sin estudiar su evolución en el tiempo.

Se recomienda proponer un programa de fortalecimiento y promoción de habilidades sociales para los adolescentes, especialmente en aquellas donde se obtuvieron los menores puntajes. Esto podría contribuir a una mejora en este tipo de habilidades y sería útil en el proceso de formación integral de los estudiantes. La capacitación al personal docente y a los padres de familia sobre la importancia de las habilidades sociales en los adolescentes sería una actividad indispensable dentro del plan de intervención.

Si bien el individuo construye su autoconcepto a lo largo del desarrollo, sería importante que las escuelas implemente los siguientes principios para facilitar este proceso: (1) la gestión de las emociones para generar una conexión entre docente-estudiante y (2) actividades del Coaching ontológico para que cada adolescente revise, y cree nuevas posibilidades y conexiones consigo mismo y con los demás. Fortalecer los conocimientos de los profesores sobre el autoconcepto en adolescentes sería una acción esencial para completar una propuesta psicopedagogía integral.

Desde el punto de vista metodológico, los investigadores recomiendan promover el estudio de estas variables en distintos escenarios sociales, de esta forma se podrá revisar su comportamiento según el contexto, condición socioeconómica, zona geográfica, etc. Como no se reportaron diferencias significativas en las habilidades sociales, el autoconcepto y la autoestima según la edad, sería importante promover investigaciones de diseño experimental-longitudinal para estudiar los posibles cambios que sufren las variables a lo largo del recorrido académico en la secundaria. Lo anterior se complementaría con estudios comparativos que evalúan su comportamiento en estudiantes de primaria, secundaria y universitarios.

\section{Referencias}

Adarve, M., Zurita, F., Gómez, V., Padial, R., \& Lara, A. (2019). Influencia de la práctica de actividad física en el autoconcepto de adolescentes. Retos, 36(36), 342-347. 
Alvarez, K. (2017). Acoso escolar y habilidades sociales en adolescentes de dos instituciones educativas estatales de Ate. Avances en Psicología, 24(2), 205-215.

http://oi.org/10.33539/avpsicol.2016.v24n2.155

Andrade-Salazar, J., Duffay-Pretel, L., Ortega-Maya, P., Ramirez-Avilés, E., \& Carvajal-Valencia, J. (2017). Autoestima y desesperanza en adolescentes de una institución educativa del Quindío. Duazary: Revista Internacional de Ciencias de la Salud, 14(2), 179 - 187. http://doi.org/10.21676/2389783X.1968

Aqeel, S. (2017). Self-efficacy and its relationship with social skills and the quality of decision-making among the students of Prince Sattam Bin Abdul-Aziz University. International Education Studies, 10(7), 108117. http://doi.org/10.5539/ies.v10n7p108

Atienza, F., Moreno, Y., \& Balaguer, I. (2000). Análisis de la dimensionalidad de la escala de autoestima de Rosenberg en una muestra de adolescentes valencianos. Revista de Psicología, 22(1-2), 29-42.

Ayvar, H. (2017). La autoestima y la asertividad en adolescentes de educación secundaria de un colegio estatal y particular de un sector del distrito de Santa Anita. Avances en Psicología, 24(2), 193-203. https://doi.org/10.33539/avpsicol.2016.v24n2.154

Ballester, L., March, M., \& Orte, C. (2006). Autoconcepto, estilos de afrontamiento y conducta del alumnado universitario. Aposta. Revista de Ciencias Sociales, (27), 1-22.

http://www.apostadigital.com/revistav3/hemeroteca/brage.pdf

Baquerizo, B., \& Geraldo, E. (2016). Autoconcepto y Habilidades Sociales en los estudiantes del nivel secundario de la Institución Educativa Puerto Pizana, 2016 [tesis de licenciatura, Universidad Peruana Unión]. Repositorio Institucional.

https://repositorio.upeu.edu.pe/handle/UPEU/580

Betancourth, S., Zambrano, C., Ceballos, A., Benavides, V., \& Villota, N.

(2017). Habilidades sociales relacionadas con el proceso de comunicación en una muestra de adolescentes. Psicoespacios, 11(18), 133- 147. http://doi.org/10.25057/21452776.898

Bi, Y., Ma, L., Yuan, F., \& Zhang, B. (2015). Self-esteem, perceived stress, and gender during adolescence: interactive links to different types of 
interpersonal relationships. The Journal of Psychology, 150(1), 36-57. https://doi.org/10.1080/00223980.2014.996512

Bleidorn, W., Arslan, R., Denissen, J., Rentfrow, P., Gebauer, J., Potter, J., \& Gosling, S. (2016). Age and gender differences in self-esteem - A cross-cultural window. Journal of Personality and Social Psychology, 111(3), 396-410. https://doi.org/10.1037/pspp0000078

Caballero, S., Contini, N., Lacunza, A., Mejail, S., \& Coronel, P. (2018).

Habilidades sociales, comportamiento agresivo y contexto socioeconómico. Un estudio comparativo con adolescentes de Tucumán (Argentina). Revista Cuadernos, (53), 183-203. http://revista.fhycs.unju.edu.ar/revistacuadernos/index.php/cuadernos/ article/view/261/414

Caldera, J., Reynoso, O., Angulo, M., Cadena, A., \& Ortíz, D. (2018). Habilidades sociales y autoconcepto en estudiantes universitarios de la región Altos Sur de Jalisco, México. Escritos de Psicología, 11(3), 144-153. http://doi.org/10.5231/psy.writ.2018.3112

Cheng, H., \& Furnham, A. (2017). Early indicators of self-esteem in teenagers: Findings from a nationally representative sample. Personality and Individual Differences, 116, 139143. http://doi.org/10.1016/j.paid.2017.04.037

Contini, E. (2015). Agresividad y habilidades sociales en la adolescencia. Una aproximación conceptual. Psicodebate, 15(2), 31-54. https://doi.org/10.18682/pd.v15i2.533

Coronel, C., Levin, M., \& Mejail, S. (2011). Las habilidades sociales en adolescentes tempranos de diferentes contextos socioeconómicos. Electronic Journal of Research in Educational Psychology, 9(1), 241-262.

http://ojs.ual.es/ojs/index.php/EJREP/article/view/1436/1671

Coy, L., \& Martin, E. (2017). Habilidades sociales y comunicativas a través del arte en jóvenes con trastorno de estrés autista. Revista Estudios Pedagógicos, 43(2), 47-64.

http://dx.doi.org/10.4067/S0718-07052017000200003

Dávila, R. (2018). Programa de actividades lúdicas para desarrollar habilidades sociales. Revista Hacedor, 2(1), 77-87.

http://revistas.uss.edu.pe/index.php/HACEDOR/article/view/979/839

Espejo, T., Zurita, F., Chacón, R., Castro, M., Martínez-Martínez, A., \& 
Pérez-Cortés, A. (2018). Actividad física y autoconcepto: dos factores de estudio en adolescentes de zona rural. Revista Iberoamericana de Psicología del Ejercicio y el Deporte, 13(2), 203-210. https://dialnet.unirioja.es/servlet/articulo? codigo $=6543402$

Estrada, E., Mamani, H., \& Arrunátegui, R. (2019). Clima social escolar y autoestima de los estudiantes de educación secundaria de la Institución Educativa “Aplicación Nuestra Señora del Rosario" de Puerto Maldonado. Perspectiva, 20(4), 388-397.

https://doi.org/10.33198/rp.v20i2.00052

Gámez-Medina, M., Guzmán-Facundo, F., Ahumada-Cortez, J., AlonsoCastillo, M., \& Gherardi-Donato, E. (2017). Autoestima y consumo de alcohol en adolescentes escolarizados. NURE Investigación, 14(88), $1-9$.

https://www.nureinvestigacion.es/OJS/index.php/nure/article/view/88 $5 / 767$

García, B. (2001). Cuestionario de Autoconcepto General CAG. Manual. Editorial EOS, S.A.

García, M., Cabanillas, G., Morán, V., \& Olaz, F. (2014). Diferencias de género en habilidades sociales en estudiantes universitarios de Argentina. Anuario electrónico de estudios en Comunicación Social "Disertaciones", 7(2), 114-135.

https://revistas.urosario.edu.co/index.php/disertaciones/article/view/37 36

García, R. (2003). Autoconcepto académico y percepción familiar. GalegoPortuguesea de psicoloxía e educación, 8(7), 359-374.

http://hdl.handle.net/2183/6953

García, S., Olivares, M., \& Racionero, F. (2017). Desarrollo de la madurez vocacional en adolescentes: difícil reto para la orientación académica y profesional. Revista de Pedagogía, 38(102), 195-216. https:/www.redalyc.org/articulo.oa?id=659/65952814010

Gatto, R., Garbin, A., Corrente, J., \& Garbin, C. (2017). Self-esteem level of Brazilian teenagers victims of bullying and its relation with the need of orthodontic treatment. RGO - Revista Gaúcha de Odontologia, 65(1), 30-36. http://doi.org/10.1590/1981863720170001000053304

Gismero, E. (2010). Manual de la Escala de Habilidades Sociales (3 $\left.3^{\mathrm{a}} \mathrm{ed}.\right)$. 
TEA Ediciones, S.A.

Góngora, V., \& Casullo, M. (2009). Validación de la escala de autoestima de Rosenberg en población general y en población clínica de la Ciudad de Buenos Aires. Revista Iberoamericana de Diagnóstico y Evaluación Psicológica, 1(27),179-194.

Guillén, F., \& Ramírez, M. (2011). Relationship between self-concept and the physical fitness of third-cycle primary school students. Revista de Psicología del Deporte, 20(1), 45-59. https://www.rpdonline.com/article/view/635/729

Gutiérrez, M., \& Expósito, J. (2015). Autoconcepto, dificultades interpersonales, habilidades sociales y conductas asertivas en adolescentes. REOP. Revista Española de Orientación y Psicopedagogía, 26(2), 42-58.

http://doi.org/10.5944/reop.vol.26.num.2.2015.15215

Harris, M., \& Orth, U. (2019). The Link Between Self-Esteem and Social Relationships: A Meta-Analysis of Longitudinal Studies. Journal of Personality and Social Psychology. Advance online publication. http://doi.org/10.1037/pspp0000265

Hernández-Sampieri, R., \& Mendoza, C. (2018). Metodología de la investigación. Las rutas cuantitativa, cualitativa y mixta. McGramHill Education.

Jaimes, C., Cruz, L., Vega, E., Balladares, P., \& Matta, H. (2019). Habilidades sociales en estudiantes adolescentes de un centro preuniversitario de Lima Norte. Health Care \& Global Health, 3(1), 39-43. http://doi.org/10.22258/hgh.2019.31.54

Jaureguizar, J., Garaigordobil, M., \& Bernaras, E. (2018). Self-concept, social skills, and resilience as moderators of the relationship between stress and childhood depression. School Mental Health, 10(4), 488499. http://doi.org/10.1007/s12310-018-9268-1

Jin-Yeul, S. (2017). The effects of interpersonal relation and social support on college freshmen's adaptation to college life. Journal of the Korea Academia-Industrial Cooperation Society, 18(12), 335-345. http://doi.org/10.5762/KAIS.2017.18.12.335

Keane, L., \& Loades, M. (2016). Review: Low self-esteem and internalizing disorders in young people - a systematic review. Child and Adolescent Mental Health, 22(1), 4-15. http://doi.org/10.1111/camh.12204 
Kipp, L. (2016). Psychosocial aspect of youth physical activity. Pediatric Exercise Science, 28(1), 28-31. http://doi.org/10.1123/pes.2016-0009

Kline, R. (2005). Principles and practice of structural equation modeling (2 ed.). The Guilford Press.

Lacunza, A., \& Contini, N. (2011). Las habilidades sociales en niños y adolescentes. Su importancia en la prevención de trastornos psicopatológicos. Fundamentos en Humanidades, 12(23), 159-182. https://ri.conicet.gov.ar/handle/11336/72163

León-Moreno, C., \& Musitu-Ferrer, D. (2019). Estilos de comunicación familiar, autoconcepto escolar y familiar, y motivación de venganza en adolescentes. European Journal of Investigation in Health, Psychology and Education, 9(1), 51-58.

https://doi.org/10.30552/ejihpe.v9i1.316

Malca-Romero, A., \& Rivera-Jiménez, L. (2019). Clima social familiar ¿Qué relación tiene con el autoconcepto en adolescentes del Callao? CASUS. Revista de Investigación y Casos en Salud, 4(2), 120129. https://doi.org/10.35626/casus.2.2019.208

Malinauskas, R., Dumciene, A., \& Lapeniene, D. (2014). Social skills and life satisfaction of lithuanian first-and senior-year university students. Social Behavior and Personality, 42(2), 285-294. http://doi.org/10.2224/sbp.2014.42.2.285

Marchant, T., Milicic, N., \& Pino, M. (2017). La autoestima en alumnos de $3^{\circ}$ a $8^{\circ}$ básico. una mirada por nivel de escolaridad y género. Revista Iberoamericana de Evaluación Educativa, 10(2), 111-125. http://dx.doi.org/10.15366/riee2017.10.2.006

Massenzana, F. (2017). Autoconcepto y autoestima: ¿sinónimos o constructos complementarios? Revista de Investigación en Psicología Social, 3(1), 39-52.

https://publicaciones.sociales.uba.ar/index.php/psicologiasocial/article /view/2336/1984

Morales, F. (2017). Relaciones entre afrontamiento del estrés cotidiano, autoconcepto, habilidades sociales e inteligencia emocional. European Journal of Education and Psychology, 10(2), 41-48. http://doi.org/10.1016/j.ejeps.2017.04.001

Moreira, E., \& Bolívar, O. (2019). Transición adolescente y su impacto en el desarrollo del pensamiento crítico y formación conductual. Revista 


\section{Tacca, Cuarez \& Quispe - Habilidades Sociales}

Cognosis, 4(5), 41-52. https://doi.org/10.33936/cognosis.v4i1.1673 Mori, M. (2018). Habilidades sociales como factores de protección del programa Adereza tu vida sin drogas ni violencia en escolares de Huaycán. PSIQUEMAG, 7(2), 73-88.

http://revistas.ucv.edu.pe/index.php/psiquemag/issue/view/228/Habili dades $\% 20$ sociales

Muñoz, V., Moreno, L., \& Jiménez, M. (2008). Las tipologías de estatus sociométrico durante la adolescencia: contraste de distintas técnicas y fórmulas para su cálculo. Psicothema, 20(4), 665-671.

https://idus.us.es/xmlui/handle/11441/56724

Núñez, C., Hernández, V., Jerez, D., Rivera, D., \& Núñez, M. (2018). Las habilidades sociales en el rendimiento académico en adolescentes. Revista de la SEECI, (47), 37-49. https://doi.org/10.15198/seeci.2018.0.37-49

Onetti, W., Fernández-García, J., \& Castillo-Rodríguez, A. (2019). Transition to middle school: Self-concept changes. PLOS ONE, 14(2). http://doi.org/10.1371/journal.pone.0212640

Orgilés, M., Samper, D., Fernández-Martínez, I., \& Espada, J. (2017). Depresión en preadolescentes españoles: Diferencias en función de variables familiares. Revista de Psicología Clínica con Niños y Adolescentes, 4(2), 129-134.

https://www.revistapcna.com/sites/default/files/17-06.pdf

Oyarzún, G., Estrada, C., Pino, E., \& Oyarzún, M. (2012). Habilidades sociales y rendimiento académico: una mirada desde el género. Acta Colombiana de Psicología, 15(2), 21-28.

http://www.scielo.org.co/pdf/acp/v15n2/v15n2a03.pdf

Patrício, M., Maia, F., \& Bezerra, C. (2015). Las habilidades sociales y el comportamiento infractor en la adolescencia. Subjetividad y Procesos Cognitivos, 19(2), 17-38.

https://www.redalyc.org/articulo.oa?id=3396/339643529001

Reynoso, O., Caldera, J., De la Torre, V., Martínez, A., \& Macías, G.

(2018). Autoconcepto y apoyo social en estudiantes de bachillerato.

Un estudio predictivo. Revista de Psicología y Ciencias del Comportamiento de la Unidad Académica de Ciencias Jurídicas y Sociales, 9(1), 100-119.

http://www.revistapcc.uat.edu.mx/index.php/RPC/article/view/207/25 
6

Riquelme, M., Garcia, O., \& Serra, E. (2018). Psychosocial maladjustment in adolescence: Parental socialization, self-esteem, and substance use. Anales de Psicología, 34(3), 536-544. https://doi.org/10.6018/analesps.34.3.315201

Ruiz, C. (2006). Test de Habilidades Sociales. Universidad Cesar Vallejo. Sadovnikova, T. (2016). Self-esteem and interpersonal relations in adolescence. Procedia - Social and Behavioral Sciences, 233, 440444. https://doi.org/10.1016/j.sbspro.2016.10.181

Santana, L., Garcés, Y., \& Feliciano, L. (2018). Incidencia del entorno en el desarrollo de habilidades sociales en adolescentes en riesgo de exclusión. Revista Electrónica de Investigación y Docencia (REID), (20), 07-22. http://doi.org/10.17561/reid.n20.1

Schoeps, K., Tamarit, A., González, R., \& Montoya-Castilla, I. (2019). Competencias emocionales y autoestima en la adolescencia: impacto sobre el ajuste psicológico. Revista de Psicología Clínica con Niños y Adolescentes, 6(1), 51-56. http://doi.org/10.21134/rpcna.2019.06.1.7

Simões, N., \& de Castro, P. (2018). Avaliação psicológica em escolares: relação entre personalidade, autoconceito e habilidades sociais. Gerais: Revista Interinstitucional de Psicologia, 11(1), 26-44. http://pepsic.bvsalud.org/scielo.php? script=sci_arttext\&pid=S198382202018000100004\&lng=pt\&tlng=pt

Tacca, D. (2018). Compendio pedagógico. Estándares ediciones.

Tacca, D. (2016). ¿Cómo aprende el que aprende? La importancia de las emociones en el aprendizaje. Revista Peruana de Psicología y Trabajo Social, 5(1), 53-65.

Tajfel, H. (1981). Human groups and social categories. Cambridge University Press.

Thompson, H., Wojciak, A., \& Cooley, M. (2016). Self-esteem: A mediator between peer relationships and behaviors of adolescents in foster care. Children and Youth Services Review, 66, 109-116. https://doi.org/10.1016/j.childyouth.2016.05.003

Veas, A., Castejón, J., Miñano, P., \& Gilar-Corbí, R. (2019). Actitudes en la adolescencia inicial y rendimiento académico: el rol mediacional del autoconcepto académico. Revista de Psicodidáctica, 24(1), 71-77. https://doi.org/10.1016/j.psicod.2018.11.001 
Viejo, C., \& Ortega-Ruiz, R. (2015). Cambios y riesgos asociados a la adolescencia. Psychology, Society \& Education, 7(5), 109-118. http://dx.doi.org/10.25115/psye.v7i2.527

Videra-García, A., \& Reigal-Garrido, R. (2013). Physical self-concept, perceptions of health and life satisfaction in a sample of adolescents. Annals of Psychology, 29(1), 141-147. https://doi.org/10.6018/analesps.29.1.132401

Daniel Rubén Tacca Huamán is a professor at the Universidad Tecnológica del Perú

Renzo Cuarez Cordero belongs to Centro preuniversitario de la Universidad Nacional Mayor de San Marcos (CEPUSM), Lima, Perú Roberto Quispe Huaycho belongs to Colegio Técnico Experimental Jesús Obrero

Contact Address: c17500@utp.edu.pe 